\title{
Cationic polymer contributes to broaden the spectrum of vancomycin activity achieving eradication of Pseudomonas aeruginosa
}

\section{Fabiana L. Alovero ( $\square$ fabiana.alovero@unc.edu.ar)}

Universidad Nacional de Cordoba Facultad de Ciencias Quimicas https://orcid.org/0000-0003-42105516

\section{Melisa B. Corti}

Universidad Nacional de Cordoba Facultad de Ciencias Quimicas

\section{Luciana P. Campagno}

Universidad Nacional de Cordoba Facultad de Ciencias Quimicas

\section{Verónica L. Romero}

Universidad Nacional de Cordoba Facultad de Ciencias Quimicas

\section{Silvina Gutierrez}

Universidad Nacional de Cordoba Facultad de Ciencias Medicas

\section{Research Article}

Keywords: Pseudomonas aeruginosa, cationic polymer, glycopeptides, outer membrane, TEM, zeta potential

Posted Date: February 28th, 2022

DOl: https://doi.org/10.21203/rs.3.rs-1385827/v1

License: (c) (i) This work is licensed under a Creative Commons Attribution 4.0 International License. Read Full License 


\section{Cationic polymer contributes to broaden the spectrum of vancomycin activity achieving eradication of Pseudomonas aeruginosa}

Melisa B. Corti ${ }^{1}$, Luciana P. Campagno ${ }^{1}$, Verónica L. Romero ${ }^{1,3}$, Silvina Gutierrez ${ }^{2}$, Fabiana L. Alovero $^{1 *}$

${ }^{1}$ Departamento de Ciencias Farmacéuticas, Facultad de Ciencias Químicas, Universidad Nacional de Córdoba and Unidad de Investigación y Desarrollo en Tecnología Farmacéutica (UNITEFA), CONICET, Córdoba, Argentina.

${ }^{2}$ Centro de Microscopia Electrónica, Facultad de Ciencias Médicas, Universidad Nacional de Córdoba, Córdoba, Argentina.

3 Present address: Instituto de Farmacología Experimental de Córdoba (IFEC), CONICET and Departamento de Farmacología, Facultad de Ciencias Químicas, Universidad Nacional de Córdoba, Córdoba, Argentina.

* Corresponding author: Fabiana L. Alovero. E-mail: fabiana.alovero@unc.edu.ar

Full postal address: Medina Allende y Haya de la Torre, Edificio Ciencias II, Ciudad Universitaria (X5000HUA), Córdoba, Argentina. Telephone: 54-351-5353865 ext. 55372. 


\begin{abstract}
Vancomycin (VAN) is unable to penetrate the outer membrane of Gram-negative bacteria and reach the target site. One approach to overcome this limitation is to associate it with compounds with permeabilizing or antimicrobial properties. EudragitE100® $(\mathrm{Eu})$ is a cationic polymer insufficiently characterized for its potential antimicrobial action. Eu-VAN combinations were characterized, the antimicrobial efficacy against Pseudomonas aeruginosa was evaluated and previous studies on the effects of Eu on bacterial envelopes were extended. Time-kill assays showed eradication of P.aeruginosa within 3-6 h exposure to Eu-VAN, whilst VAN was ineffective. Eu showed regrowth in $24 \mathrm{~h}$ and delayed colony pigmentation. Although permeabilization of bacterial envelopes or morphological alterations observed by TEM and flow cytometry after exposure to Eu were insufficient to cause bacterial death, they allowed access of VAN to the target site, since Eu-VAN/Van-FL-treated cultures showed fluorescent staining in all bacterial cells, indicating Van-FL internalization. Consequently, Eu potentiated the activity of an otherwise inactive antibiotic against $P$. aeruginosa. Moreover, Eu-VAN combinations exhibited improved physicochemical properties and could be used in the development of therapeutic alternatives in the treatment of bacterial keratitis.
\end{abstract}

\title{
Keywords
}

Pseudomonas aeruginosa; cationic polymer; glycopeptides; outer membrane; TEM; zeta potential. 


\section{Introduction}

Pseudomonas aeruginosa is a versatile Gram-negative bacterium able to survive a wide range of natural and artificial environments (de Bentzmann \& Plésiat 2011). It frequently causes infections in patients undergoing invasive procedures, individuals with debilitating disorders, and immunocompromised hosts, including burned patients. This opportunistic pathogen is also a cause of hot tub-associated infections and the leading cause of sight-threatening corneal disease in otherwise healthy contact lens wearers (Bassetti et al. 2018; Hilliam et al. 2020). Therapeutic options for $P$. aeruginosa treatment are increasingly limited due to high levels of intrinsic and acquired antibiotic resistance, with the use of different antibiotics combinations being recommended. As the discovery of new classes of antibiotics has decelerated (WHO 2017; Pang et al. 2019), the search for other treatment options is gaining increasing attention, including antimicrobial peptides, therapeutic antibodies, virulence inhibitors, antibody-drug conjugates, resistance inhibitor-antibiotic or antibiotic-potentiator combinations, efflux pump inhibitors, quorum sensing inhibitors, and antibacterial nanoparticles (Chatterjee et al. 2016; Pang 2019). Moreover, the polymer community is developing new improvements for drug delivery, by synthesizing novel antimicrobial polymers and using polymer technology to harness combination therapies (Hutnick and Pokorski 2018; Kamaruzzaman 2019; Alfei and Schito 2020). Most antimicrobial polymers are positively charged, and among these, polymers with quaternary ammonium groups are probably the most explored kind of polymeric biocides (Xue et al. 2015).

Eudragit $\mathrm{E} 100 \AA(\mathrm{Eu})$ is a cationic copolymer, based on dimethylaminoethyl methacrylate, butyl methacrylate, and methyl methacrylate, and used in the pharmaceutical field (Patra et al. 2017). Although the antimicrobial properties of numerous cationic polymers are known, Eu has not yet been fully characterized. Previously, we determined that alterations in bacterial envelopes produced by Eu in $P$. aeruginosa lead to improved efficacy of ofloxacin and ciprofloxacin against fluoroquinolone-resistant isalates (Romero 2012). Thus, due to the urgent need to develop new effective antimicrobial alternatives against $P$. aeruginosa, we extended our studies to other families of antibiotics inefficient against this pathogen. Here, we selected Vancomycin (VAN), a hydrophilic glycopeptidic antibiotic, which inhibits bacterial cell wall peptidoglycan synthesis and is used to treat infections caused by multiresistant Grampositive cocci. Due to their high molecular weight and large size, they are unable to penetrate the outer bacterial membrane of Gram-negative bacteria to reach the peptide target located in the periplasmic 
space. Therefore, these bacteria are intrinsically resistant to VAN (Nikaido and Vaara 1985; Chatterjee et al. 2016; Pang et al. 2019; Richter and Hergenrother 2019).

In this investigation, we obtained and characterized EudragitE100-Vancomycin combinations (Eu-VAN). The bactericidal action exhibited by Eu-VAN against $P$. aeruginosa was evaluated and further information on the effects of the polymer on bacterial envelopes that contribute to broadening the spectrum of action of this Gram-positive only compound was provided. Some physicochemical properties of the Eu-VAN combinations considered relevant for their potential application were also evaluated.

\section{Materials and methods}

\section{Preparation and characterization of Eudragit E100-Vancomycin combinations (Eu-VAN)}

Eu-VAN dispersions were prepared as previously reported (Quinteros et al. 2008; Romero et al. 2012), with slight modifications. First, Eudragit E100® (Röhm Pharma Polymers, Germany) (Eu, 3.10x10 ${ }^{-3}$ equivalents of amino group per gram) was dissolved in acetone (Anhedra, Argentine) and $1.0 \mathrm{~N} \mathrm{HCl}$ (Anhedra, Argentine) added to neutralize $50 \%$ of the amino groups to overcome solubility limitations. The solvent was evaporated at room temperature, with powder sieved over a 70 Mesh and stored in a tightly closed bottle and maintained in a dry place. For each experiment, the necessary amount of Eu was dissolved in a volumetric flask with a minimum volume of sterile saline solution ( $\mathrm{SSS}, \mathrm{NaCl} 0.9 \%$ ) and sonication carried out. Then, Eu-VAN combinations were obtained by adding a precisely weighed amount of Vancomycin Hydrochloride (Richet, Argentine) to Eu aqueous dispersion, adapting the concentrations of both components to those required for each experiment. After brief periods of sonication, the volume was completed. Depending on the experiment, sterile ultrapure water or SSS was used for further dilutions.

A Mettler Toledo $\mathrm{pH}$ meter determined the $\mathrm{pH}$ and a VAPRO Model 5600 Osmometer with SSS as reference $(300 \mathrm{mmol} / \mathrm{kg})$ was used for osmolarity determination. Each sample was tested in triplicate.

Filtered ultrapure water (Water Purification system, Heal Force ${ }^{\circledR}$ ) was used as solvent to obtain Eu and Eu-VAN combinations for zeta potential determinations by electrophoretic light scattering using a NanoZetasiser (Nano-ZS, Malvern $\left.{ }^{\circledR}\right)$. Each sample was tested in triplicate at $25^{\circ} \mathrm{C}$, allowing the instrument to automatically optimize the signal strength of the sample. 


\section{Bacterial strains}

P.aeruginosa ATCC 27853, P.aeruginosa FQ-R1 (a clinical isolate previously evaluated, Romero et al, 2012), and P.aeruginosa PA014 were used in this study. Bacteria were stored at $-70^{\circ} \mathrm{C}$ in Trypticase Soy Broth (Britania) supplemented with 10\% glycerol. Fresh cultures were maintained in sterile water at room temperature. Free VAN was inactive against all of the tested P.aeruginosa strains (MIC $\geq 1024 \mu \mathrm{g} / \mathrm{mL}$ ).

\section{Time-kill assays}

Due to Eu partially precipitating in culture medium over prolonged incubation periods, time-kill assays were performed in SSS supplemented with 2\% Müeller-Hinton Broth (MHB, Britania). Control tubes without treatment exhibited growth under the experimental conditions assayed, and these data were included in the figures.

P.aeruginosa overnight cultures in SSS plus 2\% MHB were diluted in fresh culture medium and incubated at $37^{\circ} \mathrm{C}$, until reaching the exponential phase of growth (approximately 3-4 $\mathrm{h}$ until 0.3 $\left.\mathrm{OD}_{625 \mathrm{~nm}}\right)$. Then, the suspension was adjusted to $\approx 0.5 \mathrm{McFarland}$ scale $\left(\approx 10^{8} \mathrm{CFU} / \mathrm{mL}\right)$ and $1 \mathrm{~mL}$ was incubated with $1 \mathrm{~mL}$ of each treatment, resulting in final concentrations: Eu-VAN combinations (250 or $1000 \mathrm{mg} / \mathrm{L}$ of polymer and $1000 \mu \mathrm{g} / \mathrm{mL}$ of VAN), VAN (1000 $\mu \mathrm{g} / \mathrm{mL})$ and drug-free Eu (250 or 1000 $\mu \mathrm{g} / \mathrm{mL}$ ). After 0 (starting), 1, 3, 6 and $24 \mathrm{~h}$ at $37^{\circ} \mathrm{C}$ in a shaker, aliquots $(0.1 \mathrm{~mL})$ were serially diluted in SSS. Diluted bacteria $(0.1 \mathrm{~mL})$ were spread on Müeller-Hinton Agar plates (MHA, Britania) in duplicate and incubated for $24 \mathrm{~h}$ at $37^{\circ} \mathrm{C}$ for colony counting $(\mathrm{CFU} / \mathrm{mL})$. Data presented are the mean of three independent experiments.

\section{Membrane zeta potential of bacterial cells}

The zeta potential of bacterial suspensions of $P$. aeruginosa $\left(\approx 10^{8} \mathrm{CFU} / \mathrm{mL}\right)$ in filtered ultrapure water or Eu-treated for $10 \mathrm{~min}$ was measured using a NanoZetasizer (Nano-ZS, Malvern ${ }^{\circledR}$ ) with detection at $90^{\circ}$ scattering angle at $37^{\circ} \mathrm{C}$. Measurements were performed at least four times for individual samples.

\section{Sensitization to detergent-induced bacteriolysis.}

To investigate the Eu-mediated sensitization of P.aeruginosa to the lytic action of the detergent SDS (0.1 and $1 \%$ ), cultures were prepared as described for time-kill assays, with the following modifications: cells were standardized to $\mathrm{Abs}_{625 \mathrm{~nm}}=0.5$ in SSS, treated with $\mathrm{Eu}$ (concentrations range: $25-1500 \mu \mathrm{g} / \mathrm{mL}$ ). After 
10 and $30 \mathrm{~min}$ at $37^{\circ} \mathrm{C}$, cultures were harvested by centrifugation, incubated with lytic agents ( 4 min at $37^{\circ} \mathrm{C}$ ) and $\mathrm{Abs}_{430 \mathrm{~nm}}$ was measured. The value of untreated control without added detergent was taken as $100 \%$; strong lysis is indicated by a low percentage. Each assay was performed at least three times. Results were analyzed using the two-tailed unpaired Student's $t$-test, with statistical significance defined as $\mathrm{p}<0.05$

\section{Recovery of Eu- treated P.aeruginosa on selective agars}

Standardized cultures, as described for time-kill assays, were added to SSS in the absence or presence of Eu $(250 \mu \mathrm{g} / \mathrm{mL})$. After $30 \mathrm{~min}$ at $37^{\circ} \mathrm{C}, 0.1 \mathrm{~mL}$ aliquots were removed, 10-fold serially diluted in SSS, and viable counts $(\mathrm{CFU} / \mathrm{mL})$ determined by spreading $0.1 \mathrm{~mL}$ of each dilution on MHA and Cetrimide Agar plates (Britania) in duplicate and incubating $24 \mathrm{~h}$ at $37^{\circ} \mathrm{C}$.

\section{Flow cytometry.}

The processed culture, as described for time-kill assays, was adjusted in SSS to $\mathrm{Abs}_{625 \mathrm{~nm}}=0.3$. Aliquots were exposed to increasing Eu concentrations (range: 150 to $2400 \mu \mathrm{g} / \mathrm{mL}$ ) and incubated at $37^{\circ} \mathrm{C}$ (untreated culture as control). Samples collected after 1, 3, 6, and $24 \mathrm{~h}$ were centrifuged (3200 g for 2 min) and washed twice with SSS. DiBAC $_{4}$ (bis-(1,3-dibutylbarbituric acid)-trimethineoxonol) (Molecular Probes, Inc., USA), a membrane potential-sensitive fluorescent dye, was added to recovering cultures (final dye concentration: $0.5 \mu \mathrm{g} / \mathrm{mL}$ ). After $5 \mathrm{~min}$ in the dark at room temperature, mixtures were acquired on a BD FACS Canto II (BD Biosciences) equipped with a 488-nm argon-ion laser. Side-scatter (SSC-A), forward-scatter (FSC-A) and fluorescence signals were collected in logarithmic scale. At least 10,000 events were recorded for each sample, and all experiments conducted in duplicate on separate days.

\section{Transmission Electron microscopy (TEM)}

A high-density bacterial suspension $(40 \mathrm{mg} / \mathrm{mL})$ was prepared from an overnight culture processed according to described for time-kill assays. Cells were incubated at $37^{\circ} \mathrm{C}$ with $\mathrm{Eu}(250 \mu \mathrm{g} / \mathrm{mL})$, VAN $(1000 \mu \mathrm{g} / \mathrm{mL})$ or Eu-VAN $(250 / 1000 \mu \mathrm{g} / \mathrm{mL})$ for $3 \mathrm{~h}$. Untreated bacterial cells served as control. Aliquots were centrifuged (3200 g - 2min), pellets washed twice with SSS, and fixed with $4 \%$ formaldehyde and $2 \%$ glutaraldehyde mixture in $0.1 \mathrm{M}$ cacodylate buffer $(2 \mathrm{~h}$ at room temperature). Cells 
were washed three times with cacodylate buffer and postfixed with $1 \%$ osmium tetra-oxide in distilled water ( $2 \mathrm{~h}$ at room temperature).Cells were dehydrated with a graded acetone series and embedded in EMbed 812 epoxy resin (Electron Microscopy Science) and polymerized for $72 \mathrm{~h}$ at $60{ }^{\circ} \mathrm{C}$. Thin sections (80-100 nm) were obtained using a JEOL Jum-7 ultramicrotome, stained with uranyl acetate/lead citrate, and analyzed on a Zeiss LEO $906 \mathrm{E}$ transmission electron microscope at an operating voltage of $80 \mathrm{kV}$. Images were captured with a MegaView III camera.

\section{Internalization of Vancomycin observed by Confocal Laser Scanning Microscopy (CLSM) and} Fluorescence Microscopy (FM)

A fluorescent BODIPY FL conjugate of vancomycin (Van-FL; TERMOFISHER) was used at a final concentration of $0.1 \mu \mathrm{g} / \mathrm{mL}$. Two $\mathrm{mL}$ bacterial suspensions, standardized as indicated in time-kill assays section were added to $2 \mathrm{~mL}$ of each treatment prepared in SSS at final concentrations: Eu-VAN/Van-FL (250 - 999.9/0.1 $\mu \mathrm{g} / \mathrm{mL})$, non-fluorescent VAN (1000 $\mu \mathrm{g} / \mathrm{mL})$, VAN/Van-FL $(999,9 / 0.1 \mu \mathrm{g} / \mathrm{mL})$ and Eu $(250 \mu \mathrm{g} / \mathrm{mL})$, and incubated at $37^{\circ} \mathrm{C}$ for $60 \mathrm{~min}$. Then, $0.5 \mathrm{~mL}$ was centrifuged $(6000 \mathrm{rpm}-5 \mathrm{~min})$, mounted on slides and viewed in a Confocal-Olympus FV1200 Microscope. The image was acquired using a 60X PLAPON (immersion in oil) lens with a numerical aperture of $1.42 \mathrm{NA}$, and a sweep over the sample with the $488 \mathrm{~nm}$ laser for the excitation of Van-FL to subsequently collect its fluorescence in the range of 500-545 nm. The acquired field was approximately $34.8 \times 34.8 \mathrm{um}^{2}$ with a pixel resolution of 34 $\mathrm{nm}$. The same laser was used to generate the transmission image with the prism, polarizer and analyzer accessories required to obtain the Differential Interference Contrast (DIC) images, which were analyzed with ImageJ software.

\section{Results}

\section{Eudragit E100 aqueous dispersions physicochemical characteristics.}

Eudragit E100 dispersions obtained were transparent, exhibited $\mathrm{pH} \geq 6.6$ and positive $\mathrm{Z}$ potential (Table

1). Eu-VAN combinations exhibited a marked increase in $\mathrm{pH}(>2.4 \mathrm{log})$ compared to free VAN solution. No significant differences were observed in osmolarity between evaluated samples. 


\section{Eu-VAN combinations lead to P.aeruginosa eradication.}

Time-kill assays were performed to assess the bactericidal activity of Eu-VAN against 3 strains of $P$. aeruginosa, with the results shown in Fig. 1a-c. Vancomycin and drug-free Eu dispersions were also evaluated for comparison purposes. Vancomycin did not exhibit bactericidal action against $P$. aeruginosa under the experimental conditions tested, while a reduction $>3 \log _{10} \mathrm{CFU} / \mathrm{mL}$ in bacterial count was observed for all strains after $1 \mathrm{~h}$ of treatment with Eu-VAN, with statistically significant differences compared to untreated control and Vancomycin-treated cultures. Eradication was evidenced between 3$6 \mathrm{~h}$ of exposure, fluctuating slightly depending on the strain and concentration of polymer evaluated in combination with Vancomycin. In preliminary studies (data not included in this article), the efficacy of Eu-VAN combinations containing the same range of concentrations of polymer but decreased concentrations of vancomycin $(125,250$ and $500 \mu \mathrm{g} / \mathrm{mL})$ were evaluated, but none exhibited eradication of $P$. aeruginosa before $24 \mathrm{~h}$ exposure. Therefore, the studies were continued with the combinations used in this report (Fig. 1). In contrast, drug-free $\mathrm{Eu}(250$ and $1000 \mu \mathrm{g} / \mathrm{mL})$ was considered bacteriostatic for all 3 strains, as these cultures showed regrowth on MHA within $24 \mathrm{~h}$, with no evidence of a $3 \log _{10}$ reduction in CFU/mL (Rawlinson et al. 2010). Furthermore, reduced pigmentation was observed compared to untreated control colonies. Pigmentation was recovered by prolonging the incubation up to 48-72 h.

\section{Eu neutralizes surface charges of P.aeruginosa}

The total charge density of polymer and bacteria cannot be measured due to a lack of suitable tools (Wilson et al. 2001; Chen et al. 2010). However, the net surface charge can be determined by measuring the zeta potential. Untreated P. aeruginosa ATCC 27853 and P. aeruginosa FQ-R1 cultures exhibited values of -31.5 and $-35.1 \mathrm{mV}$, respectively, indicating a negative cell surface charge. As expected, the Eu aqueous dispersions displayed a positive zeta potential (Table 1) due to the cationic property of the polymer. Eu-treated cultures exhibited an increase in the zeta potential, becoming positive ( 23.2 and $27.5 \mathrm{mV}$, respectively).

\section{Eu sensitizes P.aeruginosa to lysis by SDS}

Fig. 2 shows the effect of Eu on the susceptibility of $P$. aeruginosa $F Q-R 1$ to lyses in the presence of anionic detergent SDS. Exposure to the lytic agent led to a slight reduction in untreated control relative 
turbidity. In contrast, a marked lysis-promoting effect was observed for cultures previously exposed to Eu, exhibiting a polymer concentration-dependent effect. Significant differences in the lytic effect were observed between SDS concentrations, which increased with longer exposure time (data not shown).

\section{Eu sensitizes $P$.aeruginosa to Cetyl trimethyl ammonium bromide}

Cetyl trimethyl ammonium bromide, a quaternary ammonium cationic detergent, is the component of Cetrimide agar that allows selective isolation of P.aeruginosa, while other organisms cannot resist its germicidal activity (Lowbury and Collins 1955). Eu-treated cultures for $30 \mathrm{~min}$ exhibited a slight reduction in viable number on MHB (a non-selective medium) compared to untreated cultures (Table 2). By contrast, a reduction of $4.4 \log \mathrm{CFU} / \mathrm{mL}$ was observed in Cetrimide agar compared to untreated control in this selective medium.

\section{Eu-mediated changes on cell membrane potential and citosol architecture in P.aeruginosa.}

Fig. 3 (upper panels) shows histograms of $\mathrm{DiBAC}_{4}$ fluorescence in P.aeruginosa cultures in the absence (a) and presence of increasing Eu concentrations (b-d), analyzed by flow cytometry. Bacteria with cell membrane damage gradually lost normal membrane potential and depolarized, allowing access of the membrane potential indicator, and leading to increased fluorescence intensity. A fluorescent bacterial population was observed in Eu-treated cultures after 1h. The fluorescence intensity increased in a manner dependent on polymer concentration, evidenced by the shift of the fluorescence intensity peak towards higher values. This effect was slightly intensified with a longer exposure time (data not shown).

The lower panels in Fig. 3 show dot-plots of side scatter (SSC-A) versus forward scatter (FSC-A) of $P$. aeruginosa FQ-R1 in the absence (e) and presence of Eu (f-h). A polymer concentration-dependent increase in SSC-A signal is observed, with a notable displacement of bacterial population above the horizontal line (quadrants 2 and 3 of the graphs). This increase is indicative of alterations in the complexity, composition or internal organization of bacteria (Adan et al. 2017). The FSC-A signal, associated with bacterial size, also exhibited an increase compared to untreated control (Fig. 3e). Hence, the bacterial population located in quadrant 3 after Eu-exposure exhibited a notable increase in both dispersion parameters analyzed by flow cytometry. These modifications also increased slightly with longer exposure time (data not shown). 


\section{Eu produces morphological alterations}

The experimental conditions used to examine by TEM the effects of the polymer on $P$. aeruginosa were selected from time-kill curves, so there is reduction in viability without causing eradication of the culture. TEM micrographs of untreated bacteria revealed normal surface architecture of the bacterium with intact membranes (Fig. 4d). Exposure to Eu and Eu-VAN (Fig. 4a and b) resulted in local effects on the outer membrane, with a loss of material observed. The cytoplasmic electrodensity was heterogeous because of aggregation of cytosolic products. In contrast, cells exposed to vancomycin (Fig. 4c) exhibited unchanged cell membranes and preserved cell morphology.

Vancomycin internalization by P.aeruginosa observed by Confocal Laser Scanning Microscopy (CLSM) and fluorescence Microscopy (FM)

Confocal microscopy provides important information concerning the interaction of vancomycin with $P$. aeruginosa, depending on whether it occurred in the absence or presence of cationic polymer (Fig. 5). P.aeruginosa treated with free VAN/Van-FL did not show fluorescence, while cultures treated with EuVAN/Van-FL exhibited fluorescent spots along bacterial cells, indicating internalization of Van-FL.

\section{Discussion}

$P$. aeruginosa is one of pathogens urgently requiring the development of new antibiotics [4]. The shortage of new drugs is due in a large part to the difficulty of finding antibiotics that can penetrate and persist within Gram-negative species (Richter and Hergenrother 2019), with the effective permeability of its outer membrane to both hydrophobic antibiotics and high molecular weight hydrophilic drugs being critical. Vancomycin is a well-known antibiotic that cannot cross the outer membrane to access the target site (Nikaido and Vaara 1985; Pang et al. 2019; Richter and Hergenrother 2019). In our study, Eudragit E100-vancomycin combinations in aqueous dispersions (Eu-VAN) were prepared and characterized to provide new alternatives for overcoming the limitations of this antibiotic against $P$. aeruginosa. This approach was based on previous reports, where ionic interaction between protonated amino groups of Eu partially neutralized with hydrochloric acid and acidic or zwitterionic drugs produced complexes or dispersions with several improved properties (Quinteros et al. 2008; Romero et al. 2012; Quinteros et al. 2014). As expected, Eu-VAN combinations showed different physicochemical and biological properties than free VAN. Initially, Eu-VAN achieved P.aeruginosa eradication under experimental conditions in 
which no components exhibited antimicrobial efficacy. Improved efficacy was observed from a short time of exposure, whereas free VAN did not show any significant effect, with drug-free Eu only revealing a viable count reduction after a short exposure with regrowth within 24 hours. Furthermore, the efficacy of Eu-VAN against P.aeruginosa was not strain specific, exhibiting a similar performance against the 3 strains evaluated, and one of these being resistant to various antimicrobials (Romero et al. 2012).

Cationic polymers interact strongly and cooperatively with negatively charged biomolecules and constituents of cell membranes, revealing promising multipurpose utilities (Deka et al. 2014) Among these, polymers with quaternary ammonium groups were shown to be membrane-active agents (Xue et al. 2015) Surface charge neutralization has been explored as an antibacterial activity employed by agents acting on bacterial surface exhibiting membrane disturbing properties, being a quick, simple and inexpensive technique (Halder 2015). In agreement with previous reports (Helander et al. 1997; Helander et al. 2001; Halder et al. 2015), the interaction between the protonated amino groups of Eu and negative bacterial surfaces caused a change in zeta potential switching to a positive value. Modifications of the bacterial surface charge can trigger other alterations with physiological consequences (MacNair and Brown 2020). In a previous study (Romero et al. 2012), we demonstrated that Eu causes sensitization to detergent-induced bacteriolysis in P. aeruginosa. Here, we have shown the aforementioned effect occurs in a time-dependent and polymer concentration-dependent manner. Furthermore, the marked reduction in viable count on selective agar suggests that Eu alters the permeability of the outer membrane in P.aeruginosa, as it becomes susceptible to inhibition by Cetyl trimethyl ammonium bromide, against which $P$. aeruginosa is resistant when not subjected to conditions of stress or sublethal injury. Such alterations in bacterial envelopes also affect normal plasma membrane functions, as shown by the timedependent and polymer concentration-dependent increase in the fluorescence intensity of $\mathrm{DiBAC}_{4}$. Although depolarization is related to deterioration of essential cellular processes and decreased cell viability, such altered membrane polarization does not always lead to bacterial death. It depends on the degree in which the functioning of the cell is affected (Halder et al. 2015). This would explain the behavior of Eu observed in this study.

Alterations inside bacterial cells were also generated. The increase in granularity or aggregation in the cytosol of P.aeruginosa observed by TEM is in agreement with the results of flow cytometry, with a noticeable concentration-dependent increase in side scattering being observed (Fig. 3). A similar alteration of cytosolic compounds was reported in E.coli after exposure to broad spectrum antiseptic 
(Malanovic et al. 2020), as well as with antimicrobial peptides (Taute et al. 2015). According to Malanovic et al. (2020), these electron-dense dots most likely indicate ribosomal aggregation. Nevertheless, the increase in size observed in Fig. 3 (g-l) may be attributed to aggregation of bacterial cells by the action of the cationic polymer, although this requires elucidation.

It is worth mentioning another effect observed in P.aeruginosa, linked to the delayed pigmentation of colonies grown after $24 \mathrm{~h}$ of exposure to drug-free Eu. Pyocyanin is one of the multiple extracellular factors produced by $P$. aeruginosa, with an important role in the establishment and progression of infections. Since anti-virulence approaches currently under development have several limitations, including the lack of membrane-penetrating molecules in gram-negative microorganisms (Aleanizy et al. 2018; Hossain et al. 2020), this effect emerges as another valuable property of this polymer in combating $P$. aeruginosa, which adds to the permeabilizing action described above. Therefore, this topic deserves more specific and quantitative studies.

Although our results show alteration of the zeta potential after brief exposure to drug-free Eu, a sensitizing effect (permeabilization), membrane depolarization, morphological alterations and phenotypic changes, such effects were insufficient to cause bacterial death, with regrowth observed after prolonged exposure (Fig. 1). This is consistent with previous reports postulating that good permeabilizers do not necessarily correspond to compounds with inherent potent bactericidal activity (Khalil et al. 2008; Sánchez-Gómez et al. 2011) particularly when evaluated over long exposure times (Rawlinson et al. 2010). The behavior exhibited by drug-free Eu contrasts with that of chitosan, a polycationic polymer widely used in pharmaceutical formulations, which has both membrane permeabilization properties and bactericidal action, even against $P$. aeruginosa (Aleanizy et al. 2018).

Interestingly, the permeabilizing properties exhibited by Eu, while unable to kill bacteria themselves significantly altered bacterial membranes, allowing access of vancomycin to bacterial cells, as seen in the fluorescence microscopy images of P.aeruginosa culture treated with Eu-VAN plus Van-FL. These events led to a time-dependent reduction in viable count, with subsequent eradication of this Gramnegative bacterium under the experimental conditions evaluated. Consequently, Eu potentiated the activity of an otherwise inactive antibiotic against P.aeruginosa. It should be noted that when a permeabilizing agent is combined with an antibiotic, its effectiveness is not always improved. For example, Polyethylenimine shows a synergistic effect with several antibiotics against P.aeruginosa, with some exceptions such as Vancomycin (Khalil et al. 2008). Another report concluded that large-scale 
membrane disruption and permeabilization mediated by antimicrobial peptides is insufficient to make them act synergistically with vancomycin or other antibiotics (He et al. 2015). Therefore, no generalization can be established and each drug-cationic compound combination must be evaluated, with it also being necessary to analyze peculiarities of each bacterial species. In this regard, the so-called eNTRy rules generated from E. coli data could be applied to other Gram-negative ESKAPE pathogens that are also difficult to penetrate, excluding $P$. aeruginosa, which requires specific studies (Muñoz and Hergenrother 2021), highlighting the importance of the results of this study on P. aeruginosa.

Alternative proposals such as co-administration of colistin, an antibiotic with permeabilizing properties, and vancomycin, also showed improved efficacy against $P$. aeruginosa, although the risk of developing resistance to one or both drugs is a limitation of its use (Armengol et al. 2019). This aspect has not yet been evaluated in cultures treated with Eu-VAN, but there are good expectations regarding this combination, since polymers containing quaternary ammonium are less susceptible to the development of resistance by bacteria (Kumar et al. 2016).

In conclusion, the results of this study constitute an interesting proposal to the challenge of finding alternatives that can penetrate and persist in Gram-negative species. The cationic polymer Eu exerts a permeabilizing effect on the outer membrane of $P$. aeruginosa, such that its combination with vancomycin (Eu-VAN) allows this hydrophilic high molecular weight antibiotic to reach its site of action and exhibit a bactericidal effect with subsequent eradication of an intrinsically resistant pathogen. Furthermore, a small contribution is made to the challenge of developing strategies to broaden the spectrum of action of the so-called Gram-positive-only compounds (Richter and Hergenrother 2019).

Recently, a similar approach was published using short synthetic peptides to achieve a permeabilization effect in $P$. aeruginosa, and these combinations have been proposed for future application in the treatment of bacterial keratitis in long-term contact lens wearers (Hossain et al. 2020), which coincides with the interests of our laboratory. The potential use of Eu-VAN in the design of ophthalmic formulations is supported by previous studies showing good biocompatibility of a formulation containing Eu, which was harmless to rabbit eye tissues (Quinteros et al. 2014). Integrating current results with those obtained in rabbit cornea generates useful information for achieving an optimal balance, allowing the selection of polymer concentrations to design effective ophthalmic formulations with permeabilization of $P$. aeruginosa cells without affecting other cells. 
In addition to improved antimicrobial properties, Eu-VAN dispersions exhibit osmolarity values (Table 1) close to the optimal ones for ophthalmic formulations (Quinteros et al. 2014; Mohammed et al. 2019), and a higher $\mathrm{pH}$ compared to free Vancomycin. These characteristics may help reduce patient discomfort and also delay degradation of VAN, a limiting factor for formulations (Antipas et al. 1994; Imperiale et al. 2018). Regarding $\mathrm{pH}$ effects, preliminary results of ongoing studies in our laboratory are promising, increasing the possibility of using Eu-VAN in the design of effective antimicrobial pharmaceutical formulations for the treatment of keratitis, and encouraging further studies.

\section{Acknowledgments}

MC, LC and VR would like to thank CONICET- Argentina for scholarships.

The authors especially thank Dr. Paul Hobson, native speaker, for revising the manuscript. They also thank Dr. Santiago Palma for providing useful suggestions.

\section{References}

Adan A, Alizada G, Kiraz Y, Baran Y, Nalbant A (2017) Flow cytometry: basic principles and applications. Crit Rev Biotechnol 37:2, 163-76, DOI: 10.3109/07388551.2015.1128876

Aleanizy FS, Alqahtani FY, Shazly G, Alfaraj R, Alsarra I, Alshamsan A et al (2018) Measurement and evaluation of the effects of $\mathrm{pH}$ gradients on the antimicrobial and antivirulence activities of chitosan nanoparticles in Pseudomonas aeruginosa. Saudi Pharm J 26(1), 79-83.

Alfei S, Schito AM (2020) Positively Charged Polymers as Promising Devices against Multidrug Resistant Gram-Negative Bacteria: A Review. Polymers (Basel) 12(5):1195. doi: $10.3390 /$ polym 12051195

Antipas AS, Velde DV, Stella V (1994) Factors affecting the deamidation of vancomycin in aqueous solutions. Int J Pharm 109 (3): 261-69. doi.org/10.1016/0378-5173(94)90388-3

Armengol E, Domenech O, Fusté E, Pérez-Guillén I, Borrell JH, Sierra JM, Vinas M (2019) Efficacy of combinations of colistin with other antimicrobials involves membrane fluidity and efflux machinery. Infect Drug Resist 12:2031-38. doi: 10.2147/IDR.S207844

Bassetti M, Vena A, Croxatto A, Righi E, Guery B (2018) How to manage Pseudomonas aeruginosa infections. Drugs Context 29; 7: 212527. doi: 10.7573/dic.212527 
Burrows LL (2018) The Therapeutic Pipeline for Pseudomonas aeruginosa Infections. ACS Infect Dis 4(7):1041-47. doi: 10.1021/acsinfecdis.8b00112

Chatterjee M, Anju CP, Biswas L, Anil Kumar V, Gopi Mohan C, Biswas R (2016) Antibiotic resistance in Pseudomonas aeruginosa and alternative therapeutic options. Int J Med Microbiol 306(1):48-58. doi: 10.1016/j.ijmm.2015.11.004

Chen LC, Kung SK, Chen HH, Lin SB (2010) Evaluation of zeta potential difference as an indicator for antibacterial strength of low molecular weight chitosan. Carbohydr Polym 82: 913-919. doi: 10.1016/j.carbpol.2010.06.017

de Bentzmann S, Plésiat P (2011) The Pseudomonas aeruginosa opportunistic pathogen and human infections. Environ Microbiol 13(7):1655-65. doi: 10.1111/j.1462-2920.2011.02469.x.

Deka SR, Sharma AK, Kumar P (2014) Cationic polymers and their self-assembly for antibacterial applications. Curr Top Med Chem 15(13):1179-95. doi: 10.2174/1568026615666150330110602

Halder S, Yadav KK, Sarkar R, Mukherjee S, Saha P, Haldar S, Karmakar S, Sen T (2015) Alteration of Zeta potential and membrane permeability in bacteria: a study with cationic agents. Springer Plus 4:672. doi: $10.1186 / \mathrm{s} 40064-015-1476-7$

He J, Starr CG, Wimley WC (2015) A lack of synergy between membrane-permeabilizing cationic antimicrobial peptides and conventional antibiotics. Biochim Biophys Acta 1848(1 Pt A):8-15. doi: 10.1016/j.bbamem.2014.09.010

Helander IM, Alakomi HL, Latva-Kala K, Koski P (1997) Polyethyleneimine is an effective permeabilizer of gram-negative bacteria. Microbiol 143:3193-99. doi: 10.1099/00221287-143-10-3193

Helander IM, Nurmiaho-Lassila EL, Ahvenainen R, Rhoades J, Roller S (2001) Chitosan disrupts the barrier properties of the outer membrane of gram-negative bacteria. Int J Food Microbiol 71:235-44. doi: $10.1016 / \mathrm{s} 0168-1605(01) 00609-2$

Hilliam Y, Kaye S, Winstanley C (2020) Pseudomonas aeruginosa and microbial keratitis. J Med Microbiol 69(1):3-13. doi: 10.1099/jmm.0.001110

Hossain MA, Sattenapally N, Parikh HI, Li W, Rumbaugh KP, German NA (2020) Design, synthesis, and evaluation of compounds capable of reducing Pseudomonas aeruginosa virulence. Eur J Med Chem 185:111800. doi: 10.1016/j.ejmech.2019.111800

Hutnick MA, Pokorski JK (2018) Polymeric Interventions for Microbial Infections: A Review. Mol Pharm 15(8):2910-21. doi: 10.1021/acs.molpharmaceut.8b00342 
Imperiale JC, Acosta GB, Sosnik A (2018) Polymer-based carriers for ophthalmic drug delivery. J Control Release 285:106-41. doi: 10.1016/j.jconrel.2018.06.031

Kamaruzzaman NF, Tan LP, Hamdan RH, Choong SS, Wong WK, Gibson AJ, Chivu A, Pina MF (2019) Antimicrobial Polymers: The Potential Replacement of Existing Antibiotics? Int J Mol Sci 20(11):2747. doi: $10.3390 /$ ijms 20112747

Khalil H, Chen T, Riffon R, Wang R, Wang Z (2008) Synergy between polyethylenimine and different families of antibiotics against a resistant clinical isolate of Pseudomonas aeruginosa. Antimicrob Agents Chemother 52(5):1635-41. doi: 10.1128/AAC.01071-07

Kumar B, Mathur A, Pathak R, Sardana K, Gautam HK, Kumar P (2016) Evaluation of antimicrobial efficacy of quaternized poly[bis(2-chloroethyl)ether-alt-1,3-bis[3-(dimethylamino)propyl]urea] against targeted pathogenic and multi-drug-resistant bacteria. J Bioact Compat Polym 31(5):467-80. doi: $10.1177 / 0883911515627473$.

Lowbury EJ, Collins AG (1955) The use of a new cetrimide product in a selective medium for Pseudomonas pyocyanea. J Clin Pathol 8(1):47-8. doi: 10.1136/jcp.8.1.47

MacNair CR, Brown ED (2020) Outer Membrane Disruption Overcomes Intrinsic, Acquired, and Spontaneous Antibiotic Resistance. mBio 11(5):e01615-20. doi: 10.1128/mBio.01615-20

Malanovic N, Ön A, Pabst G, Zellner A, Lohner K (2020) Octenidine: Novel insights into the detailed killing mechanism of Gram-negative bacteria at a cellular and molecular level. Int J Antimicrob Agents 56(5):106146. doi: 10.1016/j.ijantimicag.2020.106146

Mohammed I, Said DG, Nubile M, Mastropasqua L, Dua HS (2019) Cathelicidin-Derived Synthetic Peptide Improves Therapeutic Potential of Vancomycin Against Pseudomonas aeruginosa. Front Microbiol 10:2190. doi: 10.3389/fmicb.2019.02190

Muñoz KA, Hergenrother PJ (2021) Facilitating Compound Entry as a Means to Discover Antibiotics for Gram-Negative Bacteria. Acc Chem Res 54(6):1322-33. doi:10.1021/acs.accounts.0c00895

Nikaido H, Vaara M (1985) Molecular basis of bacterial outer membrane permeability. Microbiol Rev 49(1):1-32.

Pang Z, Raudonis R, Glick BR, Lin TJ, Cheng Z (2019) Antibiotic resistance in Pseudomonas aeruginosa: mechanisms and alternative therapeutic strategies. Biotechnol Adv 37(1):177-92. doi: 10.1016/j.biotechadv.2018.11.013 
Patra Ch. N, Priya R, Swain S, Jena GK, Panigrahi KC, Ghose D (2017) Pharmaceutical significance of Eudragit: A review. Fut J Pharm Sci 3 (1); 33-45. doi:10.1016/j.fjps.2017.02.001

Quinteros DA, Rigo VR, Kairuz AF, Olivera ME, Manzo RH, Allemandi DA (2008) Interaction between a cationic polymethacrylate (Eudragit E100) and anionic drugs. Eur J Pharm Sci 33(1):72-9. doi: 10.1016/j.ejps.2007.10.002

Quinteros DA, Tártara LI, Palma SD, Manzo RH, Allemandi DA (2014) Ocular delivery of flurbiprofen based on Eudragit( $\left({ }^{\circledR}\right)$ E-flurbiprofen complex dispersed in aqueous solution: preparation, characterization, in vitro corneal penetration, and ocular irritation. J Pharm Sci 103(12):3859-68. doi: $10.1002 /$ jps. 24153

Rawlinson LA, Ryan SM, Mantovani G, Syrett JA, Haddleton DM, Brayden DJ (2010) Antibacterial effects of poly (2-(dimethylamino ethyl)methacrylate) against selected gram-positive and gram-negative bacteria. Biomacromolecules 1(2):443-53. doi: 10.1021/bm901166y

Richter MF, Hergenrother PJ (2019) The challenge of converting Gram-positive-only compounds into broad-spectrum antibiotics. Ann N Y Acad Sci 1435(1):18-38. doi: 10.1111/nyas.13598

Romero VL, Pons P, Bocco JL, Manzo RH, Alovero FL (2012) Eudragit E100® potentiates the bactericidal action of ofloxacin against fluoroquinolone-resistant Pseudomonas aeruginosa. FEMS Microbiol Lett 334(2):102-10. doi: 10.1111/j.1574-6968.2012.02626.x

Sánchez-Gómez S, Japelj B, Jerala R, Moriyón I, Fernández Alonso M, Leiva J, Blondelle SE, Andrä J, Brandenburg K, Lohner K, Martínez de Tejada G (2011) Structural features governing the activity of lactoferricin-derived peptides that act in synergy with antibiotics against Pseudomonas aeruginosa in vitro and in vivo. Antimicrob Agents Chemother 55(1):218-28. doi: 10.1128/AAC.00904-10

Taute H, Bester MJ, Neitz AWH, Gaspar ARM (2015) Investigation into the mechanism of action of the antimicrobial peptides Os and Os-C derived from a tick defensin. Peptides 71:179-87. doi: 10.1016/j.peptides.2015.07.017

Wilson WW, Wade MM, Holman SC, Champlin FR (2001) Status of methods for assessing bacterial cell surface charge properties based on zeta potential measurements. J Microbiol Methods 43(3):153-64. doi: $10.1016 / \mathrm{s} 0167-7012(00) 00224-4$

WHO world health organization. WHO publishes list of bacteria for which new antibiotics are urgently needed. WHO. Available from: 
https://www.who.int/news/item/27-02-2017-who-publishes-list-of-bacteria-for-which-new-antibioticsare-urgently-needed. Accessed June, 2021.

Xue Y, Xiao H, Zhang Y (2015) Antimicrobial polymeric materials with quaternary ammonium and phosphonium salts. Int J Mol Sci 16(2):3626-55. doi: 10.3390/ijms16023626

\section{Statements \& Declarations}

\section{Funding}

This work was supported by grants from Secretaria de Ciencia y Tecnología- Universidad Nacional de Córdoba (Res $\mathrm{N}^{\circ}$ 411/18); and FONCyT-Agencia Nacional de Promoción de la Investigación, Desarrollo Tecnológico e Innovación (PICT2012-0173).

Competing interest The authors have no relevant financial or non-financial interests to disclose

Ethics approval Non-applicable.

Consent to participate Non-applicable.

Consent for publication Non-applicable.

Authors' Contributions

MC, LC and VR designed the protocols, carried out the experimental part and analyzed data. SG contributed in TEM image acquisition and analysis. MC drafted the manuscript with FA. FA conceived the study, acquired funding and resources, supervised the design of experiments and data interpretation, and completed the writing of the manuscript.

\section{Data availability}

The datasets used and/or analyzed during the current study are available from the corresponding author on reasonable request.

\section{Figure captions}

Fig 1 Time-kill curves for (a) Pseudomonas aeruginosa FQ-R1, (b) Pseudomonas aeruginosa PA014 and (c) Pseudomonas aeruginosa ATCC 27853 in the presence of vancomycin (filled squares, $1000 \mu \mathrm{g} / \mathrm{mL}$ ); Eu (filled triangles, $250 \mu \mathrm{g} / \mathrm{mL}$ ); Eu (filled diamonds, $1000 \mu \mathrm{g} / \mathrm{mL}$ ); Eu-VAN (open triangles and dotted line, 250 and $1000 \mu \mathrm{g} / \mathrm{mL}$ for polymer and VAN, respectively); Eu-VAN (open diamonds and dotted line, 1000 and $1000 \mu \mathrm{g} / \mathrm{mL}$ for polymer and vancomycin, respectively) or untreated control (filled 
circles). The values are the mean \pm SD for at least three independent experiments. In some samples, the standard deviations are very small and therefore cannot be distinguished in the figure.

Fig. 2 Effect of increasing concentrations of Eu on sensitization of P. aeruginosa FQ-R1 to the lytic action of detergent. Cells were exposed to $\mathrm{Eu}$ for $10 \mathrm{~min}$ at room temperature, followed by exposure to SDS, and remaining turbidity was measured. The value of the control (without Eu or detergent) was taken as $100 \%$; strong lysis is indicated by a low percentage. Each assay was performed three times. ${ }^{*} \mathrm{P}<0.05$ was considered statistically significant.

Fig. 3 Effect of Eu on $\mathrm{DiBAC}_{4}$ fluorescence (histograms in upper panels), granularity (SSC) and bacterial size (FSC) (dot-plots in lower panels) in P.aeruginosa FQ-R1 after $1 \mathrm{~h}$ exposure at increasing polymer concentrations: (a and e) untreated control; (b-d and f-h) polymer concentrations increase from left to right: 300, 600 and $1200 \mu \mathrm{g} / \mathrm{mL}$. M1: range of undamaged cells showing no significant depolarization of cytoplasmic membrane; M2: fluorescence intensities corresponding to cells with depolarized membrane. The horizontal line in dot-plots has been positioned so that more than $95 \%$ of the untreated cells fall below the line indicating the granularity exhibited by the bacterial population unexposed to the polymer. The vertical line has been positioned so that more than $95 \%$ of the untreated cells are to the left, corresponding to the size of the bacteria without exposure to the polymer. The numbers inserted in the dot-plot (g) indicate generated quadrants, and facilitate comparative analysis of the results.

Fig. 4 Scanning electron micrographs of P.aeruginosa FQ-R1 incubated for $3 \mathrm{~h}$ with (a) Eu-VAN (250/1000 $\mu \mathrm{g} / \mathrm{mL})$, (b) Eu $(250 \mu \mathrm{g} / \mathrm{mL})$, (c) vancomycin $(1000 \mu \mathrm{g} / \mathrm{mL})$, (d) untreated control. Bacteria were photographed at a magnification of $x 46,460$.

Fig. 5 Confocal microscopic images of $P$. aeruginosa FQ-R1 incubated for $3 \mathrm{~h}$ with Eu-VAN/Van-FL (250-999.9/0.1 $\mu \mathrm{g} / \mathrm{mL}$, upper panels) or VAN/Van-FL (999.9/0.1 $\mu \mathrm{g} / \mathrm{mL}$, lower panels). (a) Bright field image, (b) fluorescence microscopy image, (c) merged images. The images were obtained with the Confocal-Olympus FV1200 microscope and analyzed with ImageJ software. 
Figures
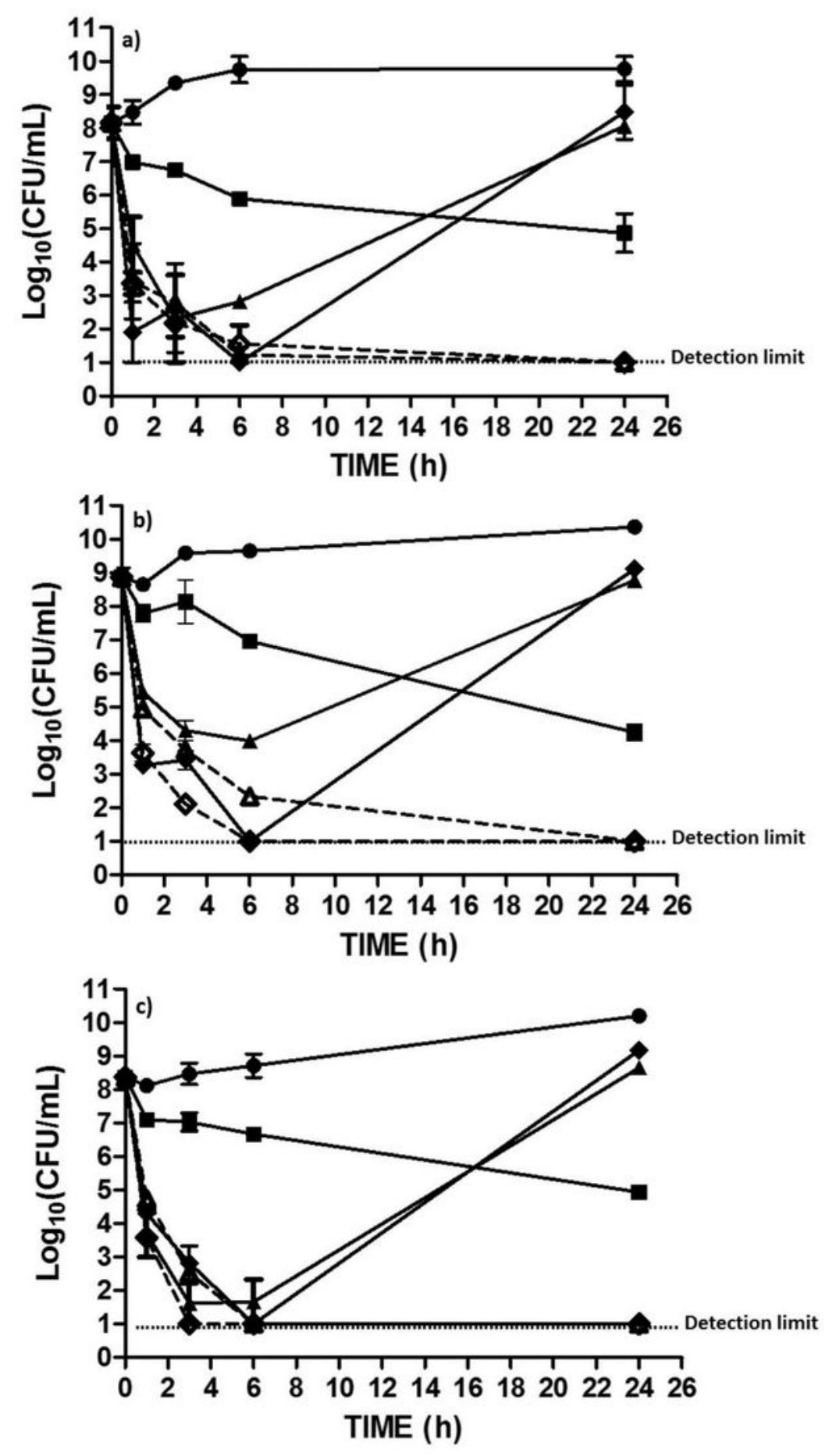

Figure 1

Time-kill curves for (a) Pseudomonas aeruginosa FQ-R1, (b) Pseudomonas aeruginosa PA014 and (c) Pseudomonas aeruginosa ATCC 27853 in the presence of vancomycin (filled squares, $1000 \mu \mathrm{g} / \mathrm{mL}$ ); Eu (filled triangles, $250 \mu \mathrm{g} / \mathrm{mL}$ ); Eu (filled diamonds, $1000 \mu \mathrm{g} / \mathrm{mL}$ ); Eu-VAN (open triangles and dotted line, 
250 and $1000 \mu \mathrm{g} / \mathrm{mL}$ for polymer and VAN, respectively); Eu-VAN (open diamonds and dotted line, 1000 and $1000 \mu \mathrm{g} / \mathrm{mL}$ for polymer and vancomycin, respectively) or untreated control (filled 19 circles). The values are the mean \pm SD for at least three independent experiments. In some samples, the standard deviations are very small and therefore cannot be distinguished in the figure.

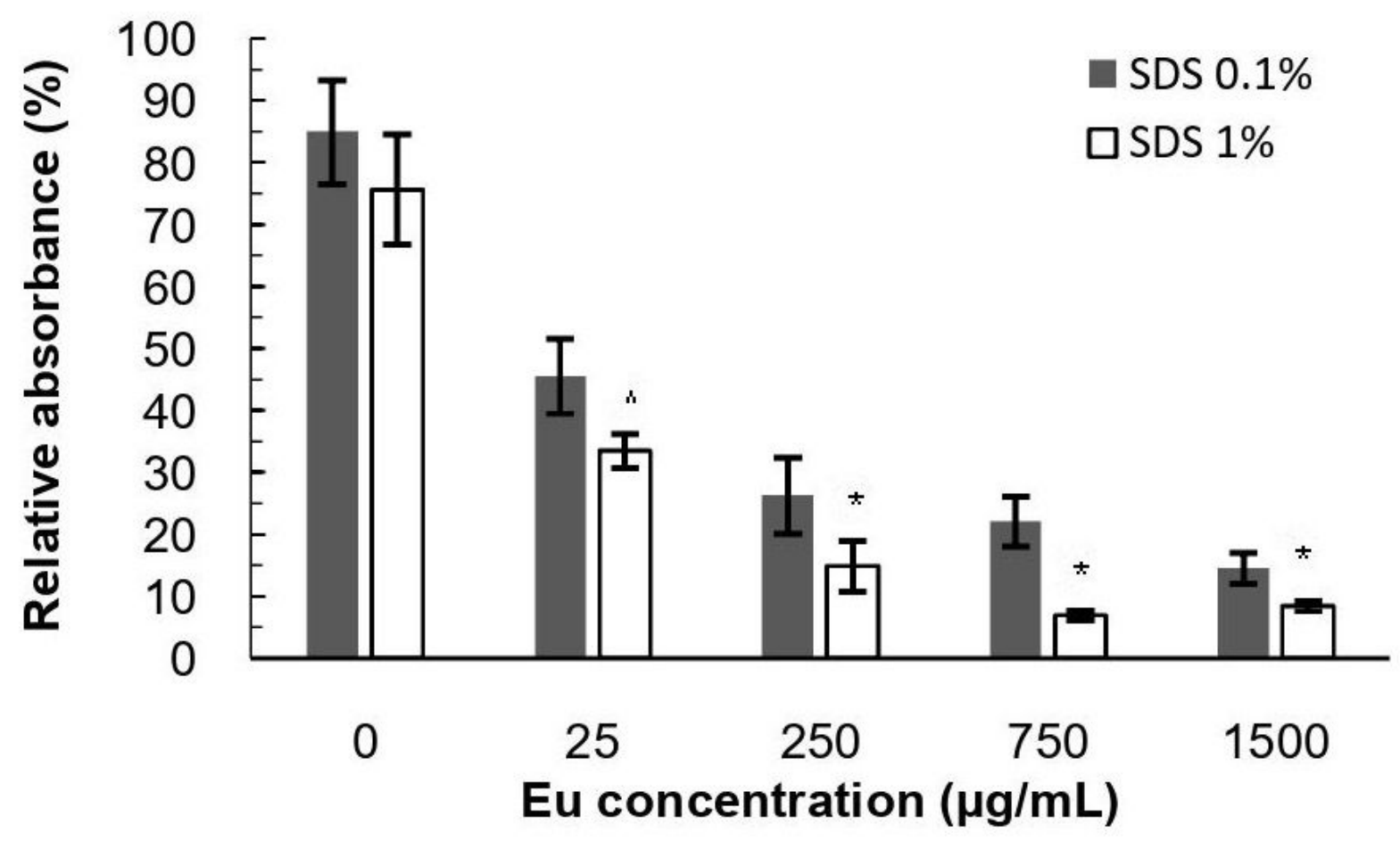

Figure 2

Effect of increasing concentrations of Eu on sensitization of P. aeruginosa FQ-R1 to the lytic action of detergent. Cells were exposed to Eu for $10 \mathrm{~min}$ at room temperature, followed by exposure to SDS, and remaining turbidity was measured. The value of the control (without Eu or detergent) was taken as $100 \%$; strong lysis is indicated by a low percentage. Each assay was performed three times. ${ }^{*} P<0.05$ was considered statistically significant. 
(a)

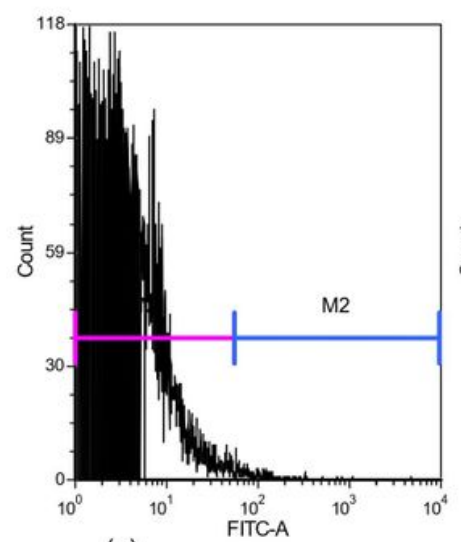

(e)

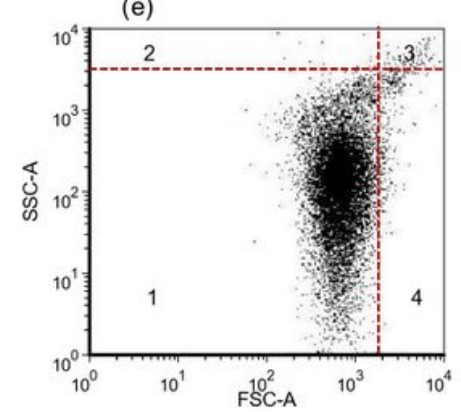

(b)
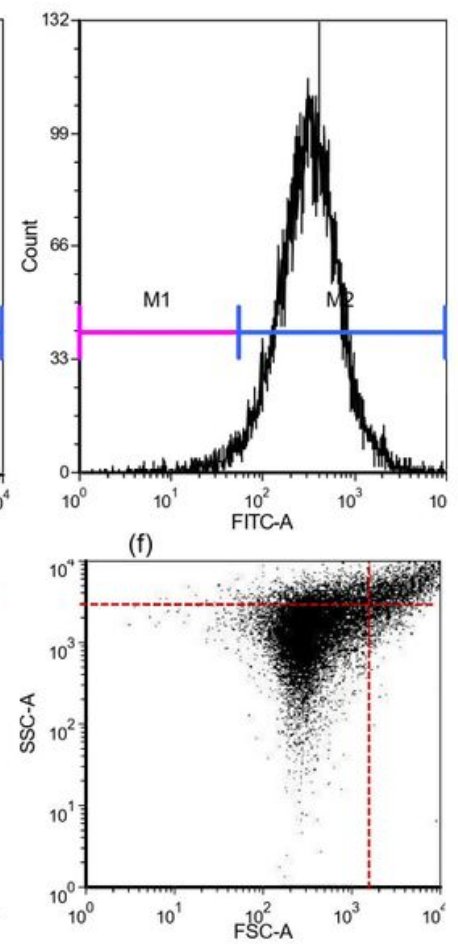

(c)

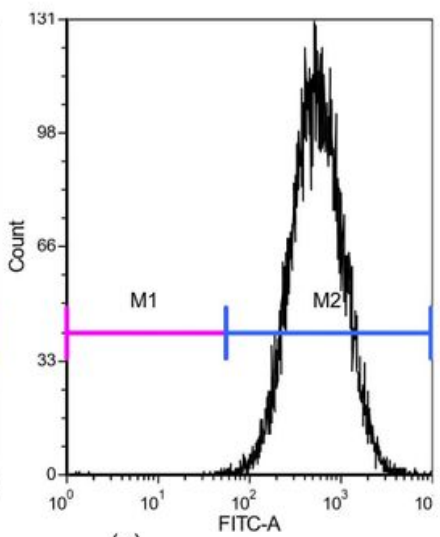

(g)

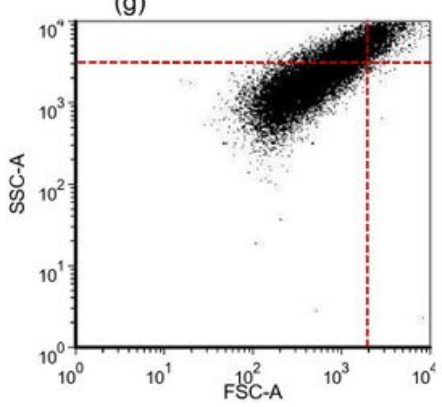

(d)

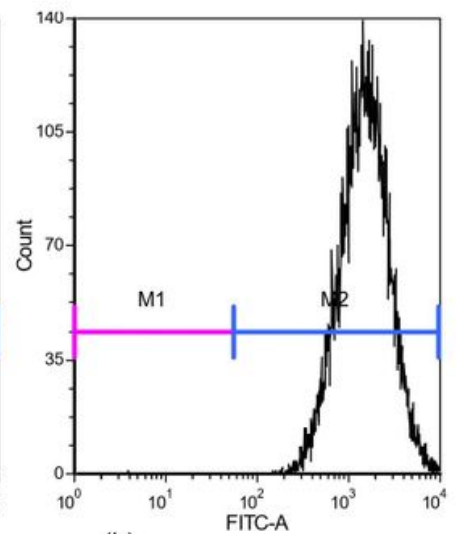

(h)

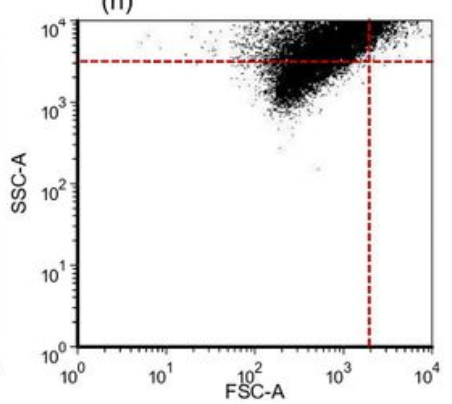

Figure 3

Effect of Eu on DiBAC4 fluorescence (histograms in upper panels), granularity (SSC) and bacterial size (FSC) (dot-plots in lower panels) in P.aeruginosa FQ-R1 after $1 \mathrm{~h}$ exposure at increasing polymer concentrations: ( $a$ and e) untreated control; ( $b-d$ and $f-h$ ) polymer concentrations increase from left to right: 300,600 and $1200 \mu \mathrm{g} / \mathrm{mL}$. M1: range of undamaged cells showing no significant depolarization of cytoplasmic membrane; M2: fluorescence intensities corresponding to cells with depolarized membrane. The horizontal line in dot-plots has been positioned so that more than $95 \%$ of the untreated cells fall below the line indicating the granularity exhibited by the bacterial population unexposed to the polymer. The vertical line has been positioned so that more than $95 \%$ of the untreated cells are to the left, corresponding to the size of the bacteria without exposure to the polymer. The numbers inserted in the dot-plot (g) indicate generated quadrants, and facilitate comparative analysis of the results. 


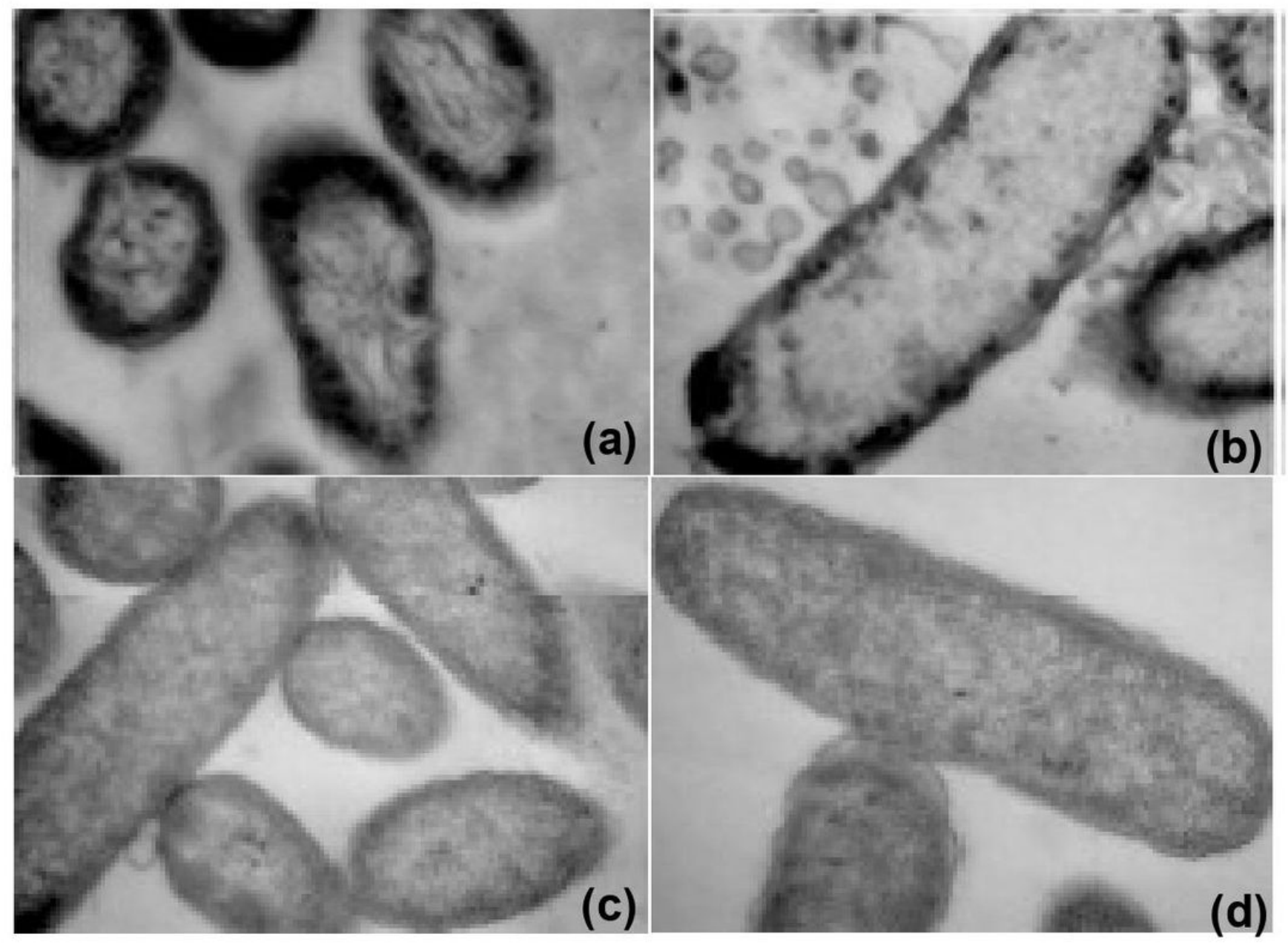

\section{Figure 4}

Scanning electron micrographs of P.aeruginosa FQ-R1 incubated for $3 \mathrm{~h}$ with (a) Eu-VAN (250/1000 $\mu \mathrm{g} / \mathrm{mL}$ ), (b) Eu $(250 \mu \mathrm{g} / \mathrm{mL})$, (c) vancomycin $(1000 \mu \mathrm{g} / \mathrm{mL})$, (d) untreated control. Bacteria were photographed at a magnification of $x 46,460$. 

a) Bright field
b) fluorescence
c) merged microscopy

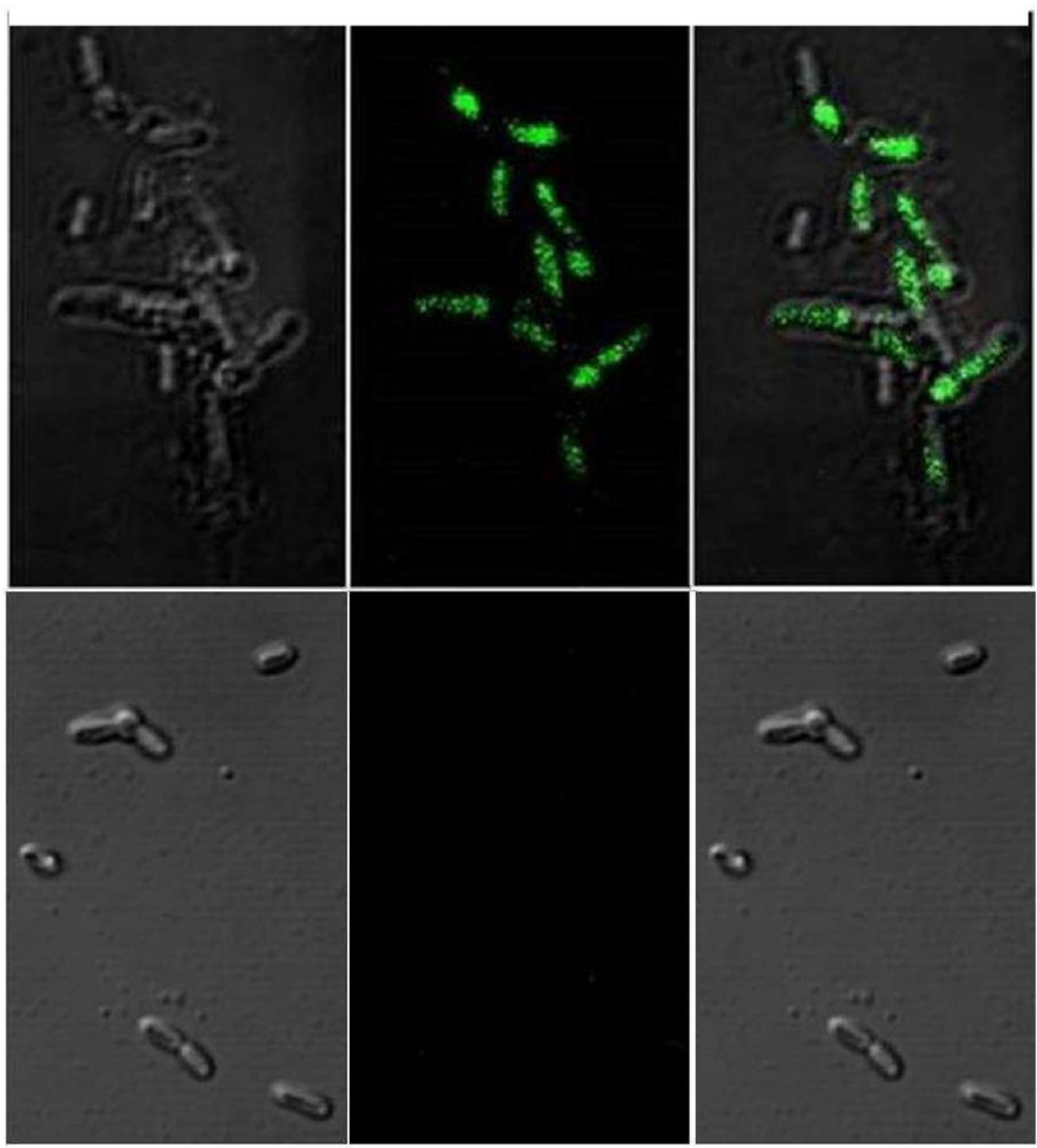

\section{Eu-VAN/VanFL}

VAN/VanFL

\section{Figure 5}

Confocal microscopic images of P. aeruginosa FQ-R1 incubated for $3 \mathrm{~h}$ with Eu-VAN/Van-FL (250999.9/0.1 $\mathrm{gg} / \mathrm{mL}$, upper panels) or VAN/Van-FL (999.9/0.1 $\mathrm{gg} / \mathrm{mL}$, lower panels). (a) Bright field image,

(b) fluorescence microscopy image, (c) merged images. The images were obtained with the ConfocalOlympus FV1200 microscope and analyzed with ImageJ software.

\section{Supplementary Files}


This is a list of supplementary files associated with this preprint. Click to download.

- Table1.pdf

- Table2.pdf 